\title{
Protocolo de prevenção do tromboembolismo venoso: Experiência de 2.000 casos em artroplastia total de joelho*
}

Venous Thromboembolism Prevention Protocol: Experience of 2,000 Cases in Total Knee Arthroplasty

\author{
Lúcio Honório de Carvalho Júnior ${ }^{1,2}$ Matheus de Almeida Correa ${ }^{1}$ Matheus Rezende Lima ${ }^{2}$ \\ Camila Barreto Silvestre $^{2}$ Victor Ferreira Almeida ${ }^{2}$ Eduardo Frois Temponi ${ }^{1,20}$ \\ ${ }^{1}$ Grupo de Joelho, Serviço de Ortopedia e Traumatologia, Hospital \\ Endereço para correspondência Eduardo Frois Temponi, MSc, \\ Madre Teresa, Belo Horizonte, MG, Brasil \\ 2 Departamento de Medicina ,Pontifícia Universidade Católica, Belo \\ Horizonte, MG, Brasil \\ Hospital Madre Teresa, Av. Raja Gabáglia 1002, Gutierrez, Belo \\ Horizonte, Minas Gerais, 30430-142, Brasil \\ (e-mail: dufrois@hotmail.com).
}

Rev Bras Ortop 2020;55(4):426-431.

\section{Resumo \\ Palavras-chave \\ - tromboembolismo/ complicações \\ - artroplastia do joelho \\ - fatores de risco}

Objetivo O objetivo do presente estudo é avaliar o impacto de um protocolo institucional em um hospital terciário na prevenção do tromboembolismo venoso em 2.005 pacientes submetidos a artroplastia total primária de joelho.

Métodos Os dados dos prontuários de pacientes submetidos a artroplastia total do joelho antes $(n=1.115)$ e após $(n=890)$ a implantação do protocolo institucional, totalizando 2.005 pacientes, foram relatados retrospectivamente. Dados demográficos, comorbidades e desfechos foram analisados.

Resultados Não houve alteração significativa nos casos de trombose venosa profunda (TVP) $(1,6 \%$ versus $2,4 \% ; p=0,211)$. Houve um aumento nos casos de embolia pulmonar (EP) $(0,2 \%$ versus $0,8 \% ; p=0,049)$.

Conclusão Apesar da implementação do protocolo de prevenção, não houve redução nos eventos estudados. A pequena incidência global faz com que novos estudos, com séries maiores, sejam necessários para confirmar ou descartar esses achados.

Objective The objective of the present study is to evaluate the impact of an institutional protocol on a tertiary hospital for the prevention of venous thromboembolism in 2005 patients submitted to primary total knee arthroplasty (TKA).

Methods Data from medical records of patients submitted TKA before $(n=1,115)$ and after $(n=890)$ the implementation of the institutional protocol, totaling 2,005 patients, were retrospectively reported. Demographics, comorbidities, and outcomes were analyzed. Results There was no significant change in the cases of deep venous thrombosis (DVT) $(1.6 \%$ versus $2.4 \% ; p=0.211)$. There was an increase in cases of pulmonary embolism (PE) $(0.2 \%$ versus $0.8 \% p=0.049)$.

Conclusion Despite the implementation of the prevention protocol, no reduction in the studied events was observed. The small global incidence makes further studies with larger series necessary to confirm or rule out these findings.

\footnotetext{
Trabalho realizado no Hospital Madre Teresa, Belo Horizonte, MG, Brasil.
}

recebido

30 de Dezembro de 2018 aceito 18 de Julho de 2019
DOI https://doi.org/ 10.1055/s-0039-1698798. ISSN 0102-3616.
Copyright $\odot 2020$ by Sociedade Brasileira License terms de Ortopedia e Traumatologia. Published by Thieme Revinter Publicações Ltda, Rio de Janeiro, Brazil 


\section{Introdução}

A artroplastia total do joelho (ATJ) é um dos procedimentos ortopédicos mais realizados em todo o mundo. ${ }^{1,2}$ Mais de 600.000 dessas cirurgias são realizadas anualmente nos Estados Unidos. ${ }^{1}$ Complicações diferentes foram descritas, e uma das mais temidas são os tromboembolismos venosos (TEVs): a trombose venosa profunda (TVP) e a embolia pulmonar (EP). ${ }^{2}$

Sem profilaxia, a TVP, sintomática ou não, poderia ser detectada em entre 41 e $84 \%$ dos exames de imagem. ${ }^{3}$ A EP não é tão comum; sua incidência varia entre 1,5 e 10\%, mas as taxas de mortalidade são de 0,1 a $1,7 \%{ }^{4}$ Muitos fatores de risco foram descritos: idade $>60$ anos, obesidade, uso de contraceptivos orais, terapia de reposição hormonal, doença inflamatória intestinal, história pessoal ou familiar de TVP ou EP, e uso prolongado de torniquete. ${ }^{5}$

Basicamente, existem métodos mecânicos e farmacológicos para prevenção da EP e da TVP. Até 2015, muitos desses métodos foram utilizados como prevenção após a ATJ em nossa instituição. Depois disso, implementamos um protocolo institucional para padronizar sua prevenção. Nossa hipótese é que, após o implante do protocolo, houve uma redução nos números de TVP e EP. O objetivo do presente estudo é verificar se este novo protocolo de padronização interferiu na incidência de TEVs após a ATJ primária.

\section{Material e Métodos}

Dados de pacientes submetidos à ATJ primária entre janeiro de 2011 e dezembro de 2017 foram coletados retrospectivamente no banco de dados da instituição, totalizando 2005 indivíduos. 0 presente estudo foi aprovado pelo comitê de ética da instituição. $\mathrm{O}$ consentimento livre e esclarecido foi obtido antes do estudo. Nenhum incentivo financeiro foi oferecido a qualquer participante. Todos os pacientes submetidos à ATJ primária foram incluídos. Os critérios de exclusão foram a realização de outro procedimento cirúrgico juntamente com a ATJ e a presença de doença infecciosa associada.

Todos os pacientes foram operados pela mesma equipe cirúrgica, com torniquete pneumático, através de abordagem anterior e artrotomia parapatelar medial. Cada ATJ foi realizada com sacrifício do ligamento cruzado posterior, e ambos os componentes foram cimentados ao mesmo tempo. 0 componente patelar não foi usado. Até dezembro de 2014, não havia um protocolo padrão sobre a profilaxia de TEV, farmacológico ou mecânico, dentro ou fora da instituição. A prevenção era executada de acordo com a orientação de cada cirurgião para o paciente. A partir de janeiro de 2015, o Hospital Madre Teresa padronizou um protocolo clínico para prevenção de TEV (-Fig. 1). Neste protocolo, cada paciente recebia $40 \mathrm{mg}$ de heparina de baixo peso molecular (HBPM) por via subcutânea, começando 6 horas após o término da cirurgia e repetida a cada 24 horas até a alta hospitalar. Nesta ocasião, a administração de anticoagulantes orais (inibidores do fator $\mathrm{Xa}$ ) era instituída e mantida até 14 dias após a cirurgia. A mesma dose de HBPM era usada a cada 12 horas caso o paciente apresentasse índice de massa corporal (IMC) $>30$. Entre as medidas mecânicas, o suporte total de peso com andador começava no primeiro dia após a recuperação do nervo periférico ou alta da unidade de terapia intensiva. $O$ tempo médio de permanência no hospital foi de 54 horas. A reabilitação doméstica e instalações de fisioterapia ambulatorial foram prescritas para cada paciente.

Os dados analisados dos pacientes foram gênero, idade, peso, estatura, classificação segundo a Sociedade Americana de Anestesiologia (ASA, na sigla em inglês), presença de diabetes, hipertensão arterial, tabagismo, tempo até a primeira caminhada, uso de anticoagulante após a alta, história de EP ou TVP e sua ocorrência até seis meses após a cirurgia. Apenas os casos sintomáticos de TEV foram analisados, bem como aqueles que necessitaram de qualquer forma de tratamento. Os casos assintomáticos não foram estudados

\section{Análise Estatística}

Os dados foram analisados usando médias e desvio padrão (DP). Os dados categóricos foram comparados usando o teste de qui-quadrado e o teste exato de Fischer. As variáveis contínuas foram submetidas à avaliação quanto à distribuição normal pelo teste de Kolmogorov-Smirnov. A diferença entre as médias foi calculada utilizando o teste paramétrico $t$ de Student e, para outras variáveis, o teste não paramétrico de Mann-Whitney. O nível de significância foi definido como 0,05 . A análise estatística foi feita em IBM SPSS Statistics for Windows, versão 20.0 (IBM Corp., Armonk, NY, EUA).

\section{Resultados}

No total, 2.005 pacientes foram analisados. Desses, 1.115 pacientes faziam parte do grupo operado antes da implantação do protocolo; 275 pacientes eram do sexo masculino $(24,7 \%)$ e $840(75,3 \%)$ do sexo feminino. A idade média foi de 72 anos. 0 peso médio foi de $78,9 \mathrm{~kg}$, e a altura média foi de $1,63 \mathrm{~m}$, com IMC médio de 29,69 . Na classificação ASA, 4,8\% dos pacientes pertenciam à classe I, $91,4 \%$ à classe II, e 3,8\% à classe III. Diabetes mellitus (DM) foi observado em $14,9 \%$ dos pacientes, e 60,9\% tinham hipertensão arterial sistêmica (HAS). A incidência de tabagismo foi de $2,5 \%$. 0 treinamento de caminhada começou em até 24 horas após a cirurgia em $85,8 \%$ dos pacientes. Nesse grupo, $44,1 \%$ utilizaram anticoagulantes por até 2 semanas após o procedimento. História de TVP ou EP estava presente em $3,8 \%$ dos casos. Trombose venosa profunda foi detectada em 1,6\% dos pacientes, e EP em 0,2\%.

O grupo operado após a implantação do protocolo era composto por 890 pacientes; 233 eram do sexo masculino $(26,2 \%)$ e 657 do sexo feminino (73,8\%). A idade média foi de 72 anos. $O$ peso médio foi de $78,3 \mathrm{~kg}$; a estatura média foi de 1,63 m, com IMC médio de 29,47. Na classificação ASA, 9,7\% pertenciam à classe I, 83,6\% à classe II, e 6,7\% à classe III. O DM estava presente em $22,7 \%$ dos pacientes, e $76,9 \%$ tinham HAS. A incidência de tabagismo foi de 5,2\%. 0 treinamento de caminhada começou em até 24 horas após a cirurgia em $78 \%$ dos pacientes. Nesse grupo, $98 \%$ utilizaram anticoagulantes por até 2 semanas após o procedimento. A história de TVP ou EP estava presente em $4,8 \%$ dos casos. Trombose venosa profunda foi detectada em $2,4 \%$ dos pacientes, e EP em 0,8\%. 


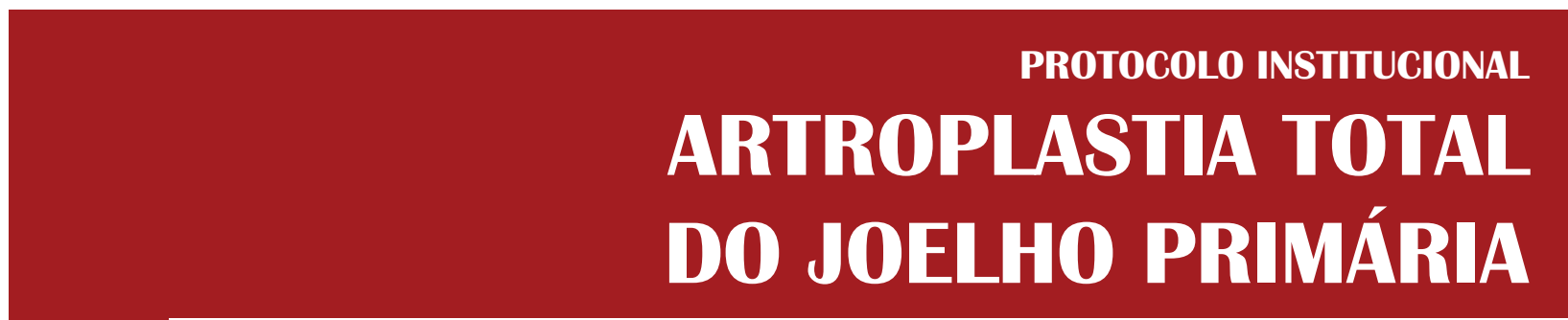

\section{INÍCIO}

Consultório Médico

Após exame clínico e radiográfico e uma vez decidido pela realização da artroplastia, solicitar exames pré-operatórios; hemograma, coagulograma, ureia, creatinina, glicemia de jejum, urina rotina, urocultura com antibiograma e a critério do cirurgião VHS e PCR. Solicitar avaliação cardiológica e consulta pré-anestésica.

Consulta com cardiologista $>$ Consulta pré-anestésica

Retorno do paciente para agendar cirurgia - Solicitar reserva de UTI quando indicado pelo anestesiologista; - Aplicar Termo de Consentimento informado e esclarecer quanto a sua obrigatoriedade;

- Orientar preparo pré-operatório: realizar banho nos $\mathbf{0 3}$ dais que antecedem a cirurgia com solução a base de clorexidine degermante e aplicação nasal de Mupirocina $2 \%$ de $12 / 12 \mathrm{~h}$ durante os $\mathbf{0 3}$ dias, conforme recomendação do $\mathrm{SCIH}$;

- Orientar a possibilidade de ingestão de $200 \mathrm{ml}$ de líquido claro adoçado (chá ou suco claro, coado e sem fibra) 02 horas antes da cirurgia;

- Emitir guia cirúrgica (incluir materiais com especificação

correta e colocar todos os códigos necessários para procedimento, registrar local a ser operado);

- Encaminhar paciente para pré-internação.

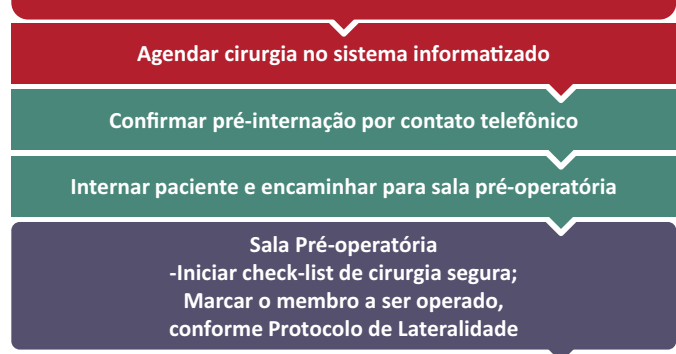

Sala Cirúrgica ou Pré-operatória

- Dar continuidade na aplicação do check-list de cirurgia segura; - Administrar antibiótico profilático conforme Kit 02 (para cirurgias com prótese) 60 minutos antes da incisão cirúrgica + repique com $2 \mathrm{G}$ de cefazolina a cada 03 horas de cirurgia; - Indução anestésica, optar por um dos esquemas: peridural com anestésico local + morfina ou peridural com anestésico local +

bloqueio femoral e isquiático ou reaquinestesia com anestésico local + morfina ou reaquinestesia com anestésico local + bloqueio femoral e isquiático ou geral + bloqueio femoral + bloqueio isquiático;

- Encaminhar paciente para sala cirúrgica, caso esteja na sala pré-anestésica.

\section{Critérios para alta hospitalar:}

- Paciente tolera deambular com auxílio de andador

Paciente com dor controlada sem necessidade de opiódes fortes

- Realizado profilaxia estendida para TEV

Realizado orientações de alta pela equipe médica

Alta hospitalar em até 72 horas
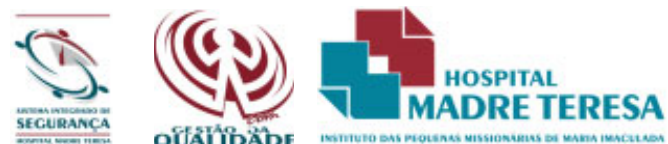

PI07V022018

\section{Sala Cirúrgica}

Realizar tonsura se necessário;

Fazer antissepsia de pele com PVPI degermante + PVPI alcoólico ou clorexidina degermante + clorexidina alcoólica. Realizar degermação durante $\mathbf{0 3}$ minutos, após fricção com solução alcoólica tintura ou clorexidina alcoólica de 01 a 02 minutos;

- Realizar cirurgia;

Ao término do procedimento, fazer RX de controle;

- Transferir paciente da mesa cirúrgica para maca e encaminhar

paciente para SRPA (Sala de Recuperação Pós-Anestésica) ou UTI.

\section{Sala de Recuperaçáo Pós-Anestésica}

- Realizar admissão pelo Enfermeiro, contemplando aplicação

Escore de Mews Cirúrgico, Gerenciamento de Risco e intervenção de Enfermagem;

Aplicar Escala de Aldret:

- Realizar cuidados com dreno, conforme recomendação do cirurgião;

- Providenciar junto a equipe de cirurgia pedido de interconsulta para clínica médica ou endocrinologia;

- Realizar avaliação médica pelo anestesiologista;

- Após avaliação da condição de alta, liberar para Unidade de Internação ou UTI.

Pós Operatório Imediato

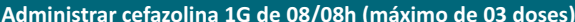
- Profilaxia TEV: HBPM SC 40mg 06 após termina da cirurgia e manter 24/24h após 1a dose. Pacientes com IMC $>30$ HBPM 40mg manutenção de $12 / 12 \mathrm{~h}$;

- Controlar a dor conforme protocolo de analgesia;

Se dreno presente, deixa-lo aberto com ou sem sucção (a critério do cirurgião) até a amanhã do dia seguinte à cirurgia;

- Manter paciente no leito sem deambular até liberação pelo médico responsável;

- Acionar clínica médica ou a endocrinologia para

acompanhamento assim que o paciente estiver no quarto.

Pós Operatório em dias subsequentes

Controlar a dor conforme protocolo de analgesia;

- Solicitar hemograma (10 dia);

- Iniciar exercícios de reabilitação e treino sob a responsabilidade da fisioterapia ou médico assistente;

Banho no 19 DPO: Cadeira de rodas ou em pé com andador, caso

seja liberado pelo médico assistente, sem molhar a ferida operatória;

- Fazer curativo no 20 DPO.

Orientações de Alta
Alta em até 72 horas
- Orientar usar medicação prescrita;
- Deambulação com andador;
- Manter ferida operatória seca após o banho;
- Realizar os exercícios recomendados dentro da tolerância;
- Realizar profilaxia estendida para TEV;
- Orienta paciente para acompanhamento ambulatorial e retorno
eventual ao Atendimento 24 Horas caso necessário.

MÉDICO INTERNAÇÃO ENFERMAGEM EQUIPE MÉDICA E ENFERMAGEM

Fig. 1 Protocolo institucional de prevenção de tromboembolismo venoso - Artroplastia total joelho. 
Tabela 1 Características dos pacientes

\begin{tabular}{|c|c|c|c|c|c|c|c|}
\hline \multicolumn{8}{|l|}{ Tabela Condensada } \\
\hline \multirow[t]{2}{*}{ Variável } & \multirow[t]{2}{*}{ Categoria } & \multicolumn{2}{|c|}{ Geral $(n=2.005)$} & \multicolumn{2}{|c|}{$\begin{array}{l}\text { Antes de } 2014 \\
(n=1.115)\end{array}$} & \multicolumn{2}{|c|}{$\begin{array}{l}\text { Depois de } 2015 \\
(n=890)\end{array}$} \\
\hline & & $\mathrm{N}$ & $\%$ & $\mathrm{~N}$ & $\%$ & $\mathrm{~N}$ & $\%$ \\
\hline \multirow[t]{3}{*}{ Sexo } & Masculino & 508 & 25,3 & 275 & 24,7 & 233 & 26,2 \\
\hline & Feminino & 1.497 & 74,7 & 840 & 75,3 & 657 & 73,8 \\
\hline & Total & 2.005 & 100,0 & 1.115 & 100,0 & 890 & 100,0 \\
\hline \multirow[t]{4}{*}{ TEV } & Sim & 9 & 0,4 & 2 & 0,2 & 7 & 0,8 \\
\hline & Não & 1.980 & 98,8 & 1.112 & 99,7 & 868 & 97,5 \\
\hline & Não informado & 16 & 0,8 & 1 & 0,1 & 15 & 1,7 \\
\hline & Total & 2.005 & 100,0 & 1.115 & 100,0 & 890 & 100,0 \\
\hline \multirow[t]{4}{*}{ TVP } & Sim & 39 & 1,9 & 18 & 1,6 & 21 & 2,4 \\
\hline & Não & 1.950 & 97,3 & 1.096 & 98,3 & 854 & 96,0 \\
\hline & Não informado & 16 & 0,8 & 1 & 0,1 & 15 & 1,7 \\
\hline & Total & 2.005 & 100,0 & 1.115 & 100,0 & 890 & 100,0 \\
\hline \multirow[t]{3}{*}{ Uso de anticoagulantes } & Sim & 1.364 & 68,0 & 492 & 44,1 & 872 & 98,0 \\
\hline & Não & 641 & 32,0 & 623 & 55,9 & 18 & 2,0 \\
\hline & Total & 2005 & 100,0 & 1.115 & 100,0 & 890 & 100,0 \\
\hline \multirow[t]{4}{*}{ HAS } & Sim & 1.294 & 64,5 & 613 & 55,0 & 681 & 76,5 \\
\hline & Não & 598 & 29,8 & 393 & 35,2 & 205 & 23,0 \\
\hline & Não informado & 113 & 5,6 & 109 & 9,8 & 4 & 0,4 \\
\hline & Total & 2.005 & 100,0 & 1.115 & 100,0 & 890 & 100,0 \\
\hline \multirow[t]{4}{*}{ DM } & Sim & 368 & 18,4 & 166 & 14,9 & 202 & 22,7 \\
\hline & Não & 1.636 & 81,6 & 949 & 85,1 & 687 & 77,2 \\
\hline & Não informado & 1 & 0,0 & 0 & 0,0 & 1 & 0,1 \\
\hline & Total & 2.005 & 100,0 & 1.115 & 100,0 & 890 & 100,0 \\
\hline \multirow[t]{3}{*}{ Tabagismo } & Sim & 74 & 3,7 & 28 & 2,5 & 46 & 5,2 \\
\hline & Não & 1.931 & 96,3 & 1.087 & 97,5 & 844 & 94,8 \\
\hline & Total & 2.005 & 100,0 & 1.115 & 100,0 & 890 & 100,0 \\
\hline \multirow[t]{4}{*}{ Histórico de TEV } & Sim & 85 & 4,2 & 42 & 3,8 & 43 & 4,8 \\
\hline & Não & 1.918 & 95,7 & 1.073 & 96,2 & 845 & 94,9 \\
\hline & Não informado & 2 & 0,1 & 0 & 0,0 & 2 & 0,2 \\
\hline & Total & 2.005 & 100,0 & 1.115 & 100,0 & 890 & 100,0 \\
\hline \multirow[t]{7}{*}{ Treinamento com caminhada } & 2 horas & 2 & 0,1 & 2 & 0,2 & 0 & 0,0 \\
\hline & 24 horas & 1.648 & 82,2 & 954 & 85,6 & 694 & 78,0 \\
\hline & 48 horas & 316 & 15,8 & 141 & 12,6 & 175 & 19,7 \\
\hline & 72 horas & 32 & 1,6 & 14 & 1,3 & 18 & 2,0 \\
\hline & 96 horas & 4 & 0,2 & 1 & 0,1 & 3 & 0,3 \\
\hline & 120 horas & 3 & 0,1 & 3 & 0,3 & 0 & 0,0 \\
\hline & Total & 2.005 & 100,0 & 1.115 & 100,0 & 890 & 100,0 \\
\hline \multirow[t]{5}{*}{ ASA } & 1 & 100 & 5,0 & 14 & 1,3 & 86 & 9,7 \\
\hline & II & 1.005 & 50,1 & 265 & 23,8 & 740 & 83,1 \\
\hline & III & 70 & 3,5 & 11 & 1,0 & 59 & 6,6 \\
\hline & Não informado & 830 & 41,4 & 825 & 74,0 & 5 & 0,6 \\
\hline & Total & 2.005 & 100,0 & 1.115 & 100,0 & 890 & 100,0 \\
\hline
\end{tabular}

Abreviações: ASA, Sociedade Americana de Anestesiologia; DM, diabetes mellitus; TVP, trombose venal profunda; HAS, hipertensão arterial sistêmica; TEV, tromboembolismo venoso. 
Comparando os resultados entre os 2 grupos, uma diferença estatística entre a classificação ASA foi encontrada $(p<0,05)$. Os casos críticos (ASA III) foram muito mais frequentes após o implante do protocolo $(p<0,05)$. Diferenças estatisticamente significativas foram encontradas entre os pacientes com DM $(p<0,05)$, HAS $(\mathrm{p}<0,05)$ e tabagistas $(\mathrm{p}<0,05)$. Depois da instituição do protocolo, essas características foram mais frequentes. Após o protocolo, o tempo até a caminhada foi significativamente tardio $(p<0,05)$. 0 uso de anticoagulantes também foi maior $(\mathrm{p}<0,05)$. Nenhuma diferença foi encontrada nos números de TVP ( $\mathrm{p}>0,05)$, e a incidência de EP foi considerada estatisticamente significativa $(p=0,049)$. Os resultados estão resumidos na - Tabela 1.

\section{Discussão}

O principal resultado deste estudo foi que, apesar da instituição deste protocolo, não houve redução nos casos de TVP, e os números de EP aumentaram. Devemos estar cientes do aumento nos números de comorbidades e da elevação significativa na utilização de cuidados intensivos no período pósoperatório. $\mathrm{O}$ atraso no treinamento de caminhada poderia explicar o aumento do número de pacientes com TEV, já que os pacientes não conseguem andar durante sua estada na unidade de terapia intensiva (UTI). A caminhada é uma forma comprovada de redução de TEV. Chandrasekaran et al. ${ }^{4}$ descreveram a incidência nula em uma população que iniciou o treinamento de caminhada nas primeiras 8 horas após o procedimento. Kjaersgaard-Andersen et al. ${ }^{6}$ descreveram uma redução de $25 \%$ nos casos de TEV quando a primeira caminhada foi iniciada em até 24 horas após a cirurgia.

Diversos estudos observaram a importante relação entre TEV e ATJ. Khokhar et al. ${ }^{3}$ descreveram 13\% de TVP e 3\% de EP. O'Reilly et al. ${ }^{7}$, analisando apenas casos sintomáticos, encontraram 0,6 a 5,7\% de TEV, 0,33 a 2,1\% de TVP, e 0 a $1 \%$ de EP. Song et al. ${ }^{8}$ realizaram flebografias bilaterais em 109 pacientes e observaram TVP sintomática em 4,6\% dos indivíduos e assintomática em 18,3\%. Sem prevenção, a incidência de TVP poderia chegar a $60 \%$ nos primeiros 90 dias após a cirurgia, ${ }^{9} \mathrm{e}$ a incidência de EP fatal poderia ser de $1,5 \%{ }^{10}$ No presente estudo, a incidência geral foi de $2,3 \%$ de TEV, com 1,6\% e $2,4 \%$ de TVP e $0,2 \%$ e $0,8 \%$ de EP pré- e pós-implante, respectivamente.

Vários fatores de risco foram relacionados à TEV. Zhang et al. ${ }^{11}$ realizaram uma meta-análise e avaliaram 1.150 .000 pacientes após artroplastias totais de joelho e de quadril. Segundo esses autores, os fatores de risco são idade $>70$ anos, sexo feminino, IMC $>30$, etnia negra, e classificação AAS $\geq 3 .^{11}$ Além de vários estudos que mostram que o protocolo de prevenção diminui a incidência de TEV, encontramos um aumento nas complicações de TEV após a ATJ. Uma explicação seria o aumento da complexidade e das comorbidades dos pacientes. Neste estudo, observamos um aumento na incidência de DM, HAS e tabagismo, embora estes fatores não estejam relacionados à maior prevalência de TEV. O pequeno número de pacientes com história prévia ou familiar de TEV poderia explicar a ausência de relação observada nos casos com TVP e EP.
Azboy et al. ${ }^{12}$, em um estudo retrospectivo com 26.415 ATJ primárias e de revisão, recomendaram a prevenção de TEV nas primeiras duas semanas após a cirurgia, pois encontraram $81 \%$ dos casos documentados ou sintomáticos de EP nos primeiros três dias após o procedimento, $89 \%$ na primeira semana e $94 \%$ nas 2 primeiras semanas. Não encontramos um protocolo de profilaxia semelhante ao utilizado nesta série. A associação entre HPBM e inibidores de $X_{a}$ não é descrita. Todos os estudos analisados usaram o mesmo fármaco durante todo o período de profilaxia.4,7,9,13 Diferentes inibidores do fator $X_{a}$ não foram considerados um fator de confusão porque, apesar dos diferentes medicamentos usados (rivaroxabana, apixabana e dabigatrana), todos têm o mesmo sítio de função e foram utilizados em doses profiláticas conforme a recomendação de fabricantes. 1

A limitação do presente estudo está em sua natureza retrospectiva, baseada no banco de dados do Hospital. A estratégia de busca usando códigos de procedimento e a leitura cuidadosa de cada prontuário tentou minimizar esse fato. Como o TEV é um evento de baixa prevalência, um período maior de acompanhamento seria necessário para verificar seu número real. Outro ponto é que o protocolo vem sofrendo alterações ao longo dos anos com a publicação de novos consensos internacionais para prevenção de EP e TEV.

\section{Conclusão}

Apesar da implantação do protocolo de prevenção, não foi observada redução nos eventos estudados. A pequena incidência global dessas doenças demanda mais estudos com maior tempo de acompanhamento para confirmar ou negar esses achados.

\section{Conflitos de Interesses}

Os autores declaram não haver conflitos de interesses.

\section{Agradecimentos}

Os autores gostariam de agradecer os demais colegas cirurgiões do Grupo de Joelho do Hospital Madre Teresa: Mateus Jacques, Tiago Jacques e Luiz Fernando Machado Soares e ao aluno da graduação em Medicina - PUC Minas João Victor Soares Asssunção.

\section{Referências}

1 HCPUnet, Healthcare Cost and Utilization Project. Agency for Healthcare Research and Quality. Disponível em: http://hcupnet.ahrq.gov

2 Ethgen $O$, Bruyère $O$, Richy F, Dardennes $C$, Reginster JY. Healthrelated quality of life in total hip and total knee arthroplasty. A qualitative and systematic review of the literature. J Bone Joint Surg Am 2004;86(05):963-974

3 Khokhar A, Chari A, Murray D, McNally M, Pandit H. Venous thromboembolism and its prophylaxis in elective knee arthroplasty: an international perspective. Knee 2013;20(03):170-176

4 Chandrasekaran S, Ariaretnam SK, Tsung J, Dickison D. Early mobilization after total knee replacement reduces the incidence of deep venous thrombosis. ANZ J Surg 2009;79(78):526-529 
5 Anderson DR, Dunbar M, Murnaghan J, et al. Aspirin or Rivaroxaban for VTE Prophylaxis after Hip or Knee Arthroplasty. N Engl J Med 2018;378(08):699-707

6 Kjaersgaard-Andersen P, Kehlet H. Should deep venous thrombosis prophylaxis be used in fast-track hip and knee replacement? Acta Orthop 2012;83(02):105-106

7 O'Reilly RF, Burgess IA, Zicat B. The prevalence of venous thromboembolism after hip and knee replacement surgery. Med J Aust 2005;182(04):154-159

8 Song $\mathrm{K}, \mathrm{Xu} \mathrm{Z}$, Rong Z, et al. The incidence of venous thromboembolism following total knee arthroplasty: a prospective study by using computed tomographic pulmonary angiography in combination with bilateral lower limb venography. Blood Coagul Fibrinolysis 2016;27(03):266-269

9 Quinlan DJ, Eikelboom JW, Dahl OE, Eriksson BI, Sidhu PS, Hirsh J. Association between asymptomatic deep vein thrombosis detected by venography and symptomatic venous thromboembolism in patients undergoing elective hip or knee surgery.J Thromb Haemost 2007;5(07):1438-1443

10 Howie C, Hughes H, Watts AC. Venous thromboembolism associated with hip and knee replacement over a ten-year period: a population-based study. J Bone Joint Surg Br 2005;87(12): $1675-1680$

11 Zhang ZH, Shen B, Yang J, Zhou ZK, Kang PD, Pei FX. Risk factors for venous thromboembolism of total hip arthroplasty and total knee arthroplasty: a systematic review of evidences in ten years. BMC Musculoskelet Disord 2015;16:24

12 Azboy I, Barrack R, Thomas AM, Haddad FS, Parvizi J. Aspirin and the prevention of venous thromboembolism following total joint arthroplasty: commonly asked questions. Bone Joint J 2017;99-B (11):1420-1430

13 Chung LH, Chen WM, Chen CF, Chen TH, Liu CL. Deep vein thrombosis after total knee arthroplasty in asian patients without prophylactic anticoagulation. Orthopedics 2011;34(01):15 